\title{
Desempeño empresarial y niveles de competitividad en Mipymes productoras de calzado en el cantón Cevallos
}

\author{
Marco Cisneros-Martínez'1, Andrea Salazar-Carranco²
}

Fecha de recepción: 4 de Mayo 2018

\section{Resumen}

Este trabajo refleja la condición de competitividad de las Mipymes productoras de calzado del cantón Cevallos basado en un estudio previo del desempeño empresarial y sus estrategias de producción. Se tomaron como referencia 15 empresas y mediante un análisis estadístico se observaron áreas como: Planificación Estratégica, Producción y Operaciones Aprovisionamiento, Logística, Aseguramiento de la Calidad, Recursos Humanos, Gestión Ambiental, Contabilidad y Auditoría. Los resultados obtenidos demostraron que su nivel de competitividad se desenvuelve localmente sin dificultad, mas no cumple con las condiciones idóneas para introducirse en el mercado internacional.

Palabras clave: Competitividad, desempeño empresarial, mipymes

\section{Abstract}

This work reflects the competitiveness condition of the SMEs that produce footwear in Cevallos. It was based on a previous study of the managerial performance and their strategies of production. There were taken 15 enterprises as a reference and through a statistical analysis it was observed areas such as: strategic planning, production and operations supply, logistics quality assurance, human resources, environmental management, accounting and auditing. The obtained results showed that their level of competitiveness gets on well with locally without any difficulty, but they don't have the appropriate conditions to get into and international market.

Keywords: Competitiveness, managerial performance, SMEs

\section{Introducción}

Establecer el desempeño empresarial de una Mipymes constituye un aspecto significativo en el marco de la idoneidad de precios, calidad y productos en mercados, más aún en contextos donde las tendencias económicas actuales a nivel internacional presionan cada día al sector empresarial a centrar sus esfuerzos en el progreso simultáneo de sus factores, procesos y sistemas de producción, que a su vez conlleven a satisfacer necesidades de mercados de mayor complejidad y con productos que ofrezcan mayor satisfaccion a los clientes. Esta dinámica se la conoce como competitividad, dentro del contexto empresarial autores como Solleiro y Castanon (2005, p. 1061) definen que:

Es la capacidad de una organización para mantener o incrementar su participación en el mercado basada en nuevas estrategias empresariales, en un sostenido crecimiento de la productividad, en la capacidad interempresarial para participar en negociaciones con diferentes instituciones y otras compañías dentro de su ambiente, determinado por el sector y el mercado de los consumidores y en políticas introducidas por los gobiernos nacionales y alianzas económicas regionales.

Montoya y Montoya (2008, p. 61). sostienen que la competitividad lleva dentro de sí la idea de indicador de calidad empresarial, de tal forma que señala como se comportan las empresas de forma individual y colectiva en los mercados y el éxito financiero y productivo de las mismas. Para lograr influencia en el mercado es necesario alcanzar niveles adecuados de productividad mediante la optimización de cada uno de los factores de producción y su correcta organización y planificación de los recursos (Cuatrecasas, 2012, p.725). en concordancia Porter (1990), sostiene que es necesario impulsar las capacidades y recursos con los que cuentan las empresas mediante un liderazgo comprometido y asertivo que al ser implementada se puede evidenciar
Fecha de aceptación: 29 de Junio 2018

el incremento de la productividad, el desarrollo de los colaboradores internos y externos, aumento del comercio y, así mismo, propicia el crecimiento local y la generación de plazas de trabajo. Otros autores apuestan a otros determinantes, tal como lo exponen Bautista y Vargas (2016), quienes indican que el factor determinante de la competitividad es la estructura empresarial porque permite el manejo ordenado de los procesos internos, que conlleva a una buena productividad. Rangel, Aguilera y Gonzales (2015) hacen incapie en la innovación, el manejo correcto de la información financiera y la protección del material intelectual empresarial mediante patentes. Por otro lado, mediante un estudio econométrico de Fuentes, Osorio y Mungaray (2016) se identifica como la clave para lograr un nivel alto de competitividad a las capacidades intangibles de las empresas, tales como el capital humano y las capacitaciones que reciben o el nivel de escolaridad puesto que estos actúan como complemento en las actividades económicas.

Según Ochoa, Parada y Verdugo (2012), la correcta gestión del conocimiento en las pequeñas y medianas empresas influye un $90 \%$ en la manera en la que se administran para conducirlas diligentemente a una visión amplia que subsecuentemente generará estrategias para buscar una capacidad distintiva o una ventaja competitiva. Por el contrario, en la ausencia de estos determinates Prokopenko (2009) sustenta que "podría llevar a la reducción de las ventas, la baja promoción, la subutilización de capacidades y la baja productividad" (p. 37).

El World Economic Forum en Global Competitiveness Report 20162017 clasifica la competitividad en tres enfoques: macroeconómico, mesoeconómico y microeconómico. Los dos primeros agrupan 12 pilares divididos en tres secciones, cuatro de requerimientos básicos, seis de potenciadores de eficiencia, y dos de factores de innovación y sofisticación. Mientras que, para medir la competitividad desde un enfoque microeconómico, Saavedra (2012) adapta 30 indicadores clasificados en 8 variables obtenidas desde un enfoque gerencial y que han sido útiles para realizar comparaciones entre empresas y de otros países.

Tabla 1. Mapa de competitividad, áreas de análisis e indicadores

\begin{tabular}{|c|c|}
\hline $\begin{array}{r}\text { Área } \\
\text { de competitividad: }\end{array}$ & Indicadores \\
\hline $\begin{array}{l}\text { Planificación } \\
\text { estratégica }\end{array}$ & $\begin{array}{l}\text { - Proceso de Planeación Estratégica } \\
\text { - Implementación de la Estrategia }\end{array}$ \\
\hline $\begin{array}{c}\text { Producción y } \\
\text { operaciones, } \\
\text { aprovisionamiento, } \\
\text { logística }\end{array}$ & $\begin{array}{l}\text { - Planificación y proceso de producción } \\
\text {-Capacidad de producción } \\
\text { - Mantenimiento } \\
\text { - Investigación y desarrollo } \\
\text { - Aprovisionamiento } \\
\text { - Manejo de inventarios } \\
\text { - Ubicación e infraestructura }\end{array}$ \\
\hline $\begin{array}{l}\text { Aseguramiento de la } \\
\text { calidad }\end{array}$ & $\begin{array}{l}\text { - Aspectos generales de la calidad } \\
\text { - Sistema de calidad }\end{array}$ \\
\hline Comercialización & $\begin{array}{l}\text { - Mercado Nacional: Mercadeo y Ventas } \\
\text { - Servicios, Distribución } \\
\text { - Mercado Exportación: Plan exportador } \\
\text { - Producto, Competencia y Mercadeo } \\
\text { - Distribución física internacional, aspectos de } \\
\text { negociación, participación en misiones y ferias }\end{array}$ \\
\hline
\end{tabular}

'Ingeniero de Empresas. Magister en Gestión de Empresas Mención Pequeñas y Medianas Empresas. Docente de la Escuela de Administración de Empresas en la Pontificia Universidad Católica del Ecuador sede Ambato. Ambato - Ecuador.E-Mail: mcisneros@pucesa.edu.ec

¿Licenciada en Ciencias Humanas y de la Educación. Magister en Educación y Currículo para la Educación Superior. Docente de la Escuela de Lenguas y Lingüística en la Pontificia Universidad Católica del Ecuador sede Ambato. Ambato - Ecuador. E-Mail: asalazar@pucesa.edu.ec 


\begin{tabular}{cl}
$\begin{array}{c}\text { Contabilidad y } \\
\text { finanzas }\end{array}$ & - Monitoreo de costos y contabilidad \\
& - Administración Financiera \\
& - Normas legales y tributarias \\
& - Aspectos generales \\
Recursos humanos & - Capacitación y promoción del personal \\
& - Cultura Organizacional \\
& - Salud y seguridad industrial \\
& - Política Ambiental de la empresa \\
& - Estrategias para proteger el medio ambiente \\
Gestión Ambiental & - Concientización y capacitación del personal \\
& en temas ambientales \\
& - Administración del Desperdicio \\
\hline Sistemas de & - Planeación del sistema \\
información & - Entradas \\
& - Procesos \\
& - Salidas
\end{tabular}

Fuente: Elaborado por Saavedra (2012)

\section{La Produccion del calzado nacional y local}

Desde un contexto nacional y según datos del Banco Central del Ecuador (2016), la influencia de la fabricación del calzado en el producto interno bruto (PIB) es del 2,20\% y su participación de la actividad económica en el valor agregado total es 0,49\%. Con respecto al aporte en el mercado laboral el Ministerio de Industrias y Productividad (2017), sostiene que el sector genera más de 100.000 plazas de trabajo de forma directa e indirecta en todo el país.

Por otra parte, entre los años 2009 y 2011, la producción por pares incrementó un 154\% como resultado de la aplicación de la política arancelaria que fue utilizada como medida de protección a pequeños productores. Esto representó un incremento en ventas internas por 318 millones de USD; es de esta manera que las Mipymes productoras de calzado del cantón Cevallos han logrado posesionarse en el mercado nacional. En lo referente a las exportaciones, los años 2013 y 2014 fueron los picos de mayores ventas con un valor promedio de 51,5 millones USD. Entre los años 2015 al 2017 la tasa de crecimiento promedio anual (TCPA) presenta una reducción de $-18,03 \%$ en valor FOB como se puede observar en la figura 1. Es decir, el calzado no ha logrado tener el mismo crecimiento en el mercado externo como lo tiene en el interno, esto debido al poco desarrollo empresarial en el sector manufacturero, como lo sostiene el Ministerio de Industrias y Productividad - MIPRO (2013).

. 93\% de las empresas solamente tuvieron ventas locales durante el último año, y un 7\% exportaron, ya sea directamente o a través de terceros.

- El potencial para exportar es únicamente del 29\% y, un 31\% desearía poder exportar a un determinado país.

. $79 \%$ de las empresas no han innovado sus procesos en los últimos dos años, y el $83 \%$ no han innovado sus productos.

. 14\% de las empresas ha participado en iniciativas de asociatividad, un $32 \%$ no está interesada en estos procesos.

- $51 \%$ de las empresas desconocen de algún programa o institución de apoyo a las exportaciones.

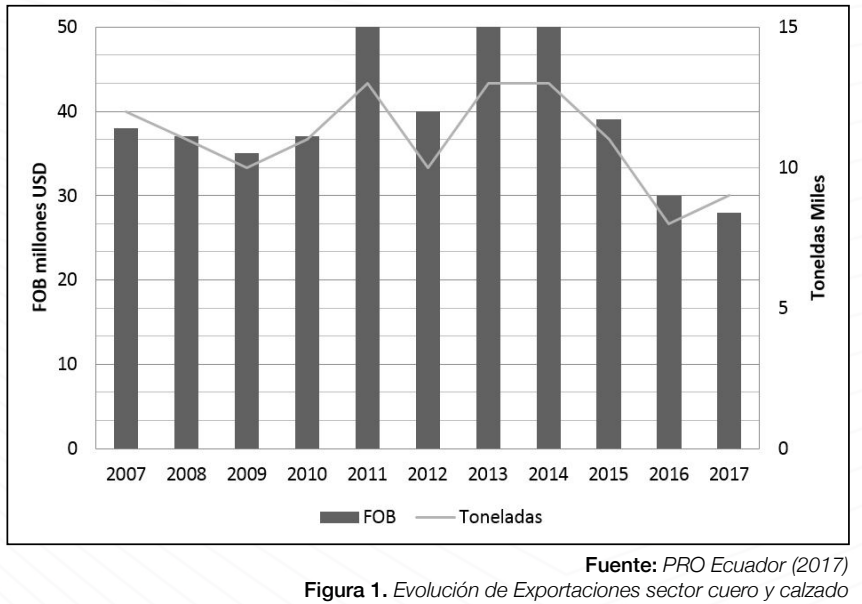

Desde un contexto local, en Cevallos la fabricación de calzado es una de las principales fuentes de ingresos de las familias, 22\% de la mano de obra se dedica a esta labor y de estos el $80 \%$ lo realiza de manera artesana. Es así como, el GAD Municipal del cantón Cevallos ha identificado las principales restricciones en cuanto a la producción:

- Existen 3 asociaciones que no agrupan a la totalidad de productores artesanales de calzado del cantón Cevallos.

- La debilidad organizativa de las asociaciones del sector impide generar una economía de escala, que refleja un débil modelo de transacción y negociación, que otorga mayor peso a sus proveedores para la compra de materias primas.

- Limitación en cuanto a diseños innovadores que impide ofertar variedad de modelos de calzado.

- Limitada capacidad de trabajar en conjunto: comprar juntos, vender juntos.

- Esquemas de comercialización que responden a iniciativas particulares o tradicionales.

- Producción no planificada territorialmente, lo que obstaculiza el mejoramiento de competencias internas.

- Dificultad para diversificar sus productos y la búsqueda organizada de acceso a líneas blandas de crédito bancario.

- La producción se caracteriza por ser únicamente bajo pedido.

- Deficiente infraestructura industrial y de equipos. (GAD-PEDC, 2009, p.33)

Por otro lado, un estudio de Bayas \& Cisneros (2013) identificó varios problemas en los productores de calzado del cantón Cevallos que resta competitividad en el mercado.

Tabla 2. Principales debilidades y amenazas de los productores de Cevallos

\section{FACTORES}

1 Utiliza procesos de fabricación exclusivos o $14 \%$ patentados

2 Tiene un programa formal de aseguramiento $4 \%$ de la calidad

3 Ha hecho inversiones significativas en $28 \%$ Equipo y/o Tecnología

$4 \quad$ Existe algún proceso donde se utiliza el $18 \%$ apoyo de otra empresa

5 Factor limitante para $\quad 5.1$ Humano-30\% la producción

\begin{tabular}{|ll|}
\hline 5.1 Humano & $30 \%$ \\
\hline 5.2 Tecnológico & $18 \%$ \\
\hline 5.3 Económico & $26 \%$ \\
\hline
\end{tabular}




\begin{tabular}{clr}
\hline $\mathbf{7}$ & $\begin{array}{l}\text { El abastecimiento brindado por sus } \\
\text { proveedores locales es suficiente para } \\
\text { abastecer su necesidad }\end{array}$ & $28 \%$ \\
\hline $\mathbf{8}$ & $\begin{array}{l}\text { Es afectado por el bajo costo de la } \\
\text { competencia }\end{array}$ & $46 \%$ \\
\hline $\mathbf{9}$ & El producto puede ser exportado & $4 \%$ \\
\hline $\mathbf{1 0}$ & $\begin{array}{l}\text { La empresa capacita al operario en } \\
\text { producción }\end{array}$ & $18 \%$ \\
\hline $\mathbf{1 1}$ & $\begin{array}{l}\text { La coordinación como asociaciones de } \\
\text { productores es eficiente }\end{array}$ & $\mathbf{2 8 \%}$ \\
\hline $\mathbf{1 2}$ & Tiene instalaciones de fabricación eficientes & $32 \%$ \\
\hline & \multicolumn{2}{c}{ Fuente: Bayas \& Cisneros (2012) }
\end{tabular}

\section{Metodología}

La presente investigación tiene un enfoque cualitativo de tipo exploratorio, puesto que se efectuó a través de un estudio descriptivo, transversal y no experimental, utilizando como datos el estudio de tesis de maestría "Análisis de la competitividad del sistema manufacturero de calzado en el cantón Cevallos" de Bayas y Cisneros (2012). Este fue referido del modelo de los determinantes de la ventaja competitiva planteado por Porter y aplicado al sector. Sin embargo, para dicho estudio se consideró las fuerzas competitivas, más no la capacidad competitiva, que influyen específicamente en los resultados empresariales de forma individual.

Los pasos metodológicos fueron los siguientes:

a) Investigación bibliográfica. Como elemento primordial para la información sobre la taxonomía de las prioridades competitivas a fin de soportar teóricamente la investigación. De igual manera, se consideró información proporcionada por instituciones gubernamentales.

b) Selección y recopilación de información de Mipymes más representativas del sector. La muestra considerada dentro de una población de 250 productores de calzado fue seleccionada a través de tres asociaciones que agrupan un total de 60 empresas. Este número fue analizado en el estudio de 2012 de Bayas y Cisneros. Posteriormente, se escogieron a las 15 Mipymes más representativas del sector en la localidad en base al promedio de ventas anuales que alcanzaron los $\$ 61.000$ para un nuevo análisis, tal como lo propone Sarache, Cardenas, Giraldo \& Parra (2007). Seguidamente, se preparó un cuestionario dividido en 7 secciones con 23 preguntas que tienen como sustento el mapa de competitividad propuesto por Saavedra (2012). Este instrumento fue aplicado a los gerentes/propietarios de las Mipymes.

c) Analizar la información recopilada mediante la correlación de dimensiones de variables de competitividad de Pearson, Hall (2015). Es una medida de la fuerza de la relación lineal entre dos variables y puede ir de -1 a 1. Donde -1 indica una relación lineal negativa perfecta entre las variables. Una $r$ de 0 indica que no hay relación lineal entre las variables, y una $r$ de 1 indica una relación lineal positiva perfecta entre las variables. De ahí que se tomó las 6 áreas de competitividad expresadas en la tabla 1 y se analizó utilizando el software estadístico SPSS, que permitió identificar los niveles de relación existentes.

\section{Resultados}

Para presentar de mejor manera los resultados, la información recopilada se divide en etapas. En la primera se evidencia la valoración estadística de las seis primeras áreas del mapa de competitividad, haciendo el análisis a partir de datos estadísticos.

\begin{tabular}{|c|c|}
\hline $\begin{array}{c}\text { Áreas de } \\
\text { Competitividad }\end{array}$ & Valoración a partir de datos de estudio \\
\hline $\begin{array}{l}\text { Planificación } \\
\text { estratégica }\end{array}$ & $\begin{array}{l}\text { Un } 33 \% \text { de las empresas conocen sobre procesos de planificación estratégica; } \\
\text { aunque su conocimiento está orientado solamente a la visión y misión, no existe } \\
\text { apertura al cambio y al trabajar en un contexto con orientación a objetivos a largo } \\
\text { plazo. Es así como solamente un } 26 \% \text { implementa en su producción alguna } \\
\text { estrategia innovadora o diferenciadora. }\end{array}$ \\
\hline $\begin{array}{l}\text { Aprovisionamiento, } \\
\text { Logística }\end{array}$ & $\begin{array}{l}\text { En esta área de competitividad se encontró que el } 66.7 \% \text { planifica su producción } \\
\text { mediante hojas de pedido, donde las cantidades son proporcionadas a discreción del } \\
\text { propietario. El inventario del producto es del } 80 \% \text {, el mismo que es manejado en } \\
\text { base al mínimo de cantidad que tiene disponible para la venta en los locales } \\
\text { comerciales. Por otra parte el } 46 \% \text { de la Mipymes realizan el mantenimiento de la } \\
\text { maquinaria de manera correctiva y muy pocas veces de forma preventiva. }\end{array}$ \\
\hline $\begin{array}{l}\text { En Aseguramiento } \\
\text { de la Calidad }\end{array}$ & $\begin{array}{l}\text { El criterio de calidad es manejado en base a criterios planteados por la propia } \\
\text { empresa. El } 60 \% \text { de productores afirman su nivel de calidad de acuerdo a sus } \\
\text { criterios y a las opiniones que el cliente expresa, el cual es el } 93 \% \text { de satisfacción. En } \\
\text { términos globales, } 95 \% \text { no aplica ninguna norma de calidad regulada por alguna } \\
\text { institución acreditadora. }\end{array}$ \\
\hline $\begin{array}{l}\text { En Recursos } \\
\text { Humanos }\end{array}$ & $\begin{array}{l}\text { La formación y actualización permanente de los operarios en base al requerimiento } \\
\text { de la producción es bajo, ya que solamente un } 20 \% \text { lo realiza de manera regular. Con } \\
\text { respecto a la salud y seguridad del operario se observa que el } 53 \% \text { de las empresas } \\
\text { se enfocan en desarrollar consciencia preventiva y hábitos de trabajo seguros } \\
\text { conforme a los objetivos planteados por el Ministerio de Relaciones Laborales a } \\
\text { través del Programa de Seguridad y Salud en el Trabajo. }\end{array}$ \\
\hline
\end{tabular}

En Gestión Respecto a políticas de control ambiental de residuos en las empresas, un $40 \%$ tiene Ambiental un control de desperdicios de la producción, mas no tiene un conocimiento especifico sobre actividades contaminantes o que causan impacto en el ambiente. De estas, el $33 \%$ realizan capacitación del personal en temas de eliminación de desperdicios. En general, las politicas ambientales son desconocidas o mal interpretadas, ya que en la totalidad consideraran que sus actividades operacionales no causan daño al entorno.

En Contabilidad y El monitoreo de control de costos incluye manejar los procesos relacionados con el Auditoría

planificar, estructurar, estimar y presupuestar los costos de modo que se logre determinar el desempeño de los recursos invertidos. Es así como el $46 \%$ de las empresas tienen dentro de su nómina una persona titular que se encarga de este proceso; el resto prefiere no tener personal en relación de dependencia y trabajan mediante la prestación de servicios profesionales.

Con respecto a la evaluación del costo operacional $60 \%$ no considera el ciclo de vida de la maquinaria, por consiguiente el valor por inactividad no es cuantificado.

Fuente: Propia en base a encuesta aplicadas (2017)

Conjuntamente se identifica que variables tienen relación entre si y cuales pierden significancia. Como se observa en la tabla 4, se concluye que la planificación estratégica no es un factor de impacto dentro las empresas estudiadas, debido a que no existe relación directa con ninguna de las áreas. Mientras que el área Producción y Operaciones Aprovisionamiento Logística mantiene una correlación significante a nivel 0.05 con 3 áreas como: los Recursos Humanos, la Gestión Ambiental y la Comercialización. Así mismo, una correlación significativa a nivel 0.01 se encuentra en las áreas de Recursos Humanos y Contabilidad y Auditoría relacionada con la Producción y Operaciones.

Tabla 4. Correlación de variables de Mipymes productores de Cevallos

\begin{tabular}{|c|c|c|c|c|c|c|c|c|}
\hline Varial & bles de control & $\begin{array}{l}\text { Planificación } \\
\text { _Estratégica }\end{array}$ & $\begin{array}{l}\text { Producción_y_y } \\
\text { operaciones_a } \\
\text { provisionami } \\
\text { ento_logística }\end{array}$ & $\begin{array}{l}\text { Aseguramiento } \\
\text { _de_la_calidad }\end{array}$ & $\begin{array}{l}\text { Recursos_- } \\
\text { Humanos }\end{array}$ & $\begin{array}{l}\text { Gestín } \\
\text { ambiental }\end{array}$ & $\begin{array}{l}\text { Contabilidad } \\
\text { y_Auditoria }\end{array}$ & Comercialización \\
\hline \multirow{4}{*}{$\begin{array}{l}\text { Planificación } \\
\text { Estratégica } \\
\text { Producción_y_op } \\
\text { eraciones_aprovi } \\
\text { sionamiento_logí }\end{array}$} & Correlación de Pearson & 1 & .357 & ,228 & ,394 & ,220 & 132 & ,484 \\
\hline & Sig. (bilateral) & & 191 & ,414 & ,146 & .432 & 639 & .067 \\
\hline & Correlación de Pearson &, 357 & 1 & $.628^{\circ}$ & $.763^{\prime \prime}$ & $.562^{\circ}$ & $.838^{\prime \prime}$ & $.606^{\circ}$ \\
\hline & Sig. (bilateral) & 191 & & .012 & ,001 & .029 & .000 & .017 \\
\hline $\begin{array}{l}\text { Aseguramiento_d } \\
\text { e_la_calidad }\end{array}$ & $\begin{array}{l}\text { Correlación de Pearson } \\
\text { Sig. (bilateral) }\end{array}$ & $\begin{array}{l}, 228 \\
414\end{array}$ & $\begin{array}{l}628^{\circ} \\
012\end{array}$ & 1 & $\begin{array}{r}.728^{*} \\
.002\end{array}$ & $\begin{array}{l}.282 \\
309\end{array}$ &, $582^{\circ}$ &, $590^{\circ}$ \\
\hline \multirow{2}{*}{$\begin{array}{l}\text { Recursos_Human } \\
\text { os }\end{array}$} & Correlación de Pearson &, 394 & $.763^{\prime \prime}$ & $.728^{\circ}$ & 1 & 260 & $684^{*}$ & $.531^{\circ}$ \\
\hline & Sig. (bilateral) & 146 &, 001 &, 002 & & 350 &, 005 & ,042 \\
\hline \multirow{2}{*}{$\begin{array}{l}\text { Gestión_Ambient } \\
\text { al }\end{array}$} & Correlación de Pearson & 220 &, $562^{\circ}$ & 282 & 260 & 1 & $629^{\circ}$ & .145 \\
\hline & Sig. (bilateral) & .432 & 029 & 309 &, 350 & & 012 & 607 \\
\hline \multirow{3}{*}{$\begin{array}{l}\text { Contabilidad_y_A } \\
\text { uditoria }\end{array}$} & Correlación de Pearson & , 132 & $838 "$ & $.582^{\circ}$ & .684" & $.629^{\circ}$ & 1 & 284 \\
\hline & Sig. (billateral) & 639 & .000 &, 023 & ,005 & 012 & & 305 \\
\hline & Correlación de Pearson & 484 & $606^{\circ}$ & $.590^{\circ}$ & $.531^{\circ}$ & 145 & 284 & 1 \\
\hline \multirow{2}{*}{\multicolumn{2}{|c|}{$\begin{array}{c}\text { Comercialización Sig. (bilateral) } \\
\text { N }\end{array}$}} & ,067 & 017 &, 021 & 042 & ,607 & ,305 & \\
\hline & & 15 & 15 & 15 & 15 & 15 & 15 & 15 \\
\hline
\end{tabular}

* La correlación es significante al nivel 0,05 (bilateral)

** La correlación es significativa al nivel 0,01 (bilateral)

Fuente: Propia en bases a encuesta aplicadas (2017) 
En lo referente al área de mercado internacional del producto, se identifica que solamente un $6 \%$ de las Mipymes exportan su producto en un promedio de 3 veces al año y su mercado principal es Colombia y Perú. Por tanto, el nivel de exposición que tiene el producto frente al comercio mundial es mínimo. Con respecto al mercado nacional se evidencia que el $86 \%$ realiza ventas internas, específicamente en locales comerciales de su propiedad y en los días de mayor afluencia comercial que son sábados y domingos. Otra parte del producto se comercializa en ciudades como Quito, Cuenca y Guayaquil una vez por semana.

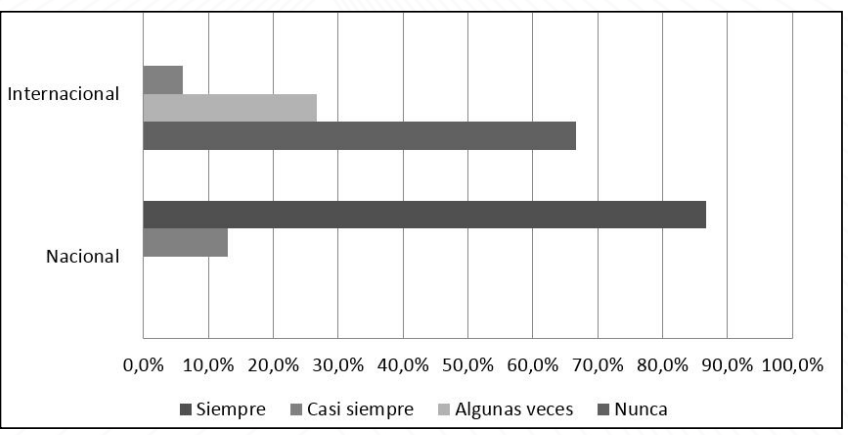

Fuente: Propia en bases a encuesta aplicadas

Figura 2. Ventas por mercado de la Mipymes

La figura 3 presenta datos con respecto a las ventas. Se recalca que la información fue levantada en dos fases: la primera en el año 2013, donde se consideró las ventas del 2009 y 2012, tomando una población de 50 empresas que son agremiadas. En la segunda fase existe una variación en la población de estudio estimando las 15 empresas más destacadas de la primera fase por su proyección comercial y situación financiera. Es necesario mencionar que 5 empresas no facilitaron información con respecto a sus ventas por motivos de discreción empresarial.

Los resultados reflejan que la media de ventas del 2012 superó los $\$ 61.000$ anuales con un precio promedio de $\$ 48$ por par de zapato, mientras que en 2017 el promedio fue de \$93.000. De todos modos, se puede manifestar que tres empresas disminuyeron sus ventas, teniendo una variación negativa $-0,487 \%$, mientras que 6 empresas incrementaron sus ventas con una variación promedio de 0,792\% y solamente una empresa se mantiene en las mismas condiciones.

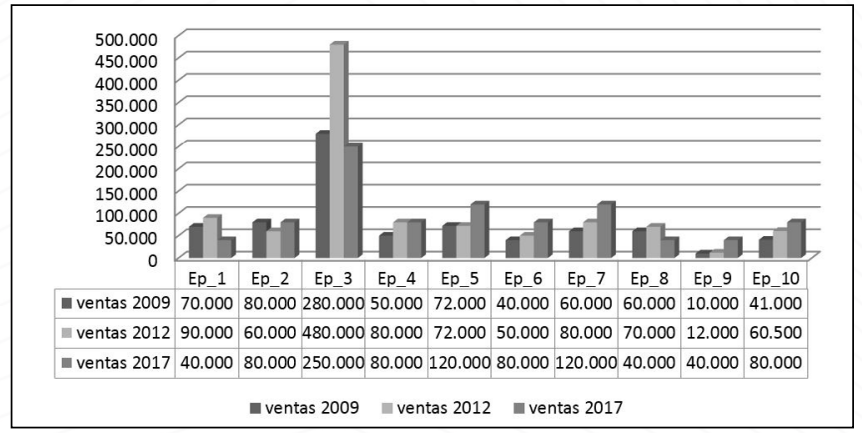

Fuente: Propia en bases a encuesta aplicadas (2012 y 2017) Figura 3. Ventas promedio por periodos de la Mipymes.

\section{Conclusiones}

El objetivo primordial del presente estudio es el de aclarar la importancia de la competitividad en las Mipymes productoras de calzado de Cevallos para expandirse comercialmente a un contexto internacional.

Es así que se llega a las siguientes conclusiones:

- Desde hace varios años, el sector productor de calzado presenta un crecimiento estable, convirtiéndose en un motivante para el emprendimiento en condiciones básicas y aceptablemente favorables. Sin embargo, esto influye en la elaboración de productos con costos no reales y conlleva a una competencia desleal. Esto implica que los produc- tores lancen al mercado calzado de precios bajos para el consumidor que a su vez perjudica a los demás empresarios del área.

- La baja capacidad de integración entre los productores conlleva a una producción manufacturera individualista, con poca productividad, con procesos manuales y lentos, escasa innovación y/o variedad en el producto. Esto se evidencia claramente en los resultados obtenidos en las áreas de Producción y Operaciones Aprovisionamiento, Logística. Así mismo la integración de los productores de calzado del cantón es meramente gremial y, de cierta manera, se la considera como un compromiso adquirido. La fuerza asociativa debería propender al crecimiento económico, empresarial y de mercado de los miembros de las tres asociaciones.

Es evidente que la planificación estratégica para los productores no representa un factor vinculante con las otras áreas de competitividad, debido a que las Mipymes manejan sus procesos de manera informal. Es por esta razón que las empresas no logran concretar la proyección internacional, ni buscan estrategias para proceder con una expansión comercial a largo plazo que les permita destacarse entre sus pares competitivos internacionales tales como Colombia y Perú.

- La favorable posición de ventas en el mercado local de las Mipymes analizadas no les permite proyectarse a un mercado internacional, considerando que el $90 \%$ de sus ventas son en el país. Esta postura conveniente ha ocasionado que los productores se centren únicamente a complacer los requerimientos de clientes locales, que no exploren las últimas tendencias globales en diseño y moda. Los productores no han logrado crear un vínculo con sus consumidores ni con la comunidad productora de calzado mediante lanzamientos públicos de nuevas colecciones para dar a conocer y recibir nuevas propuestas.

- Las áreas competitivas: Producción y Operaciones Aprovisionamiento, Logística, Aseguramiento de la Calidad, Gestión Ambiental, Contabilidad y Auditoría de alguna u otra manera, son las que tienen correlación de impacto positivo y significativo en todas sus dimensiones. A pesar de este conjunto de fortalezas altamente explotables, las empresas no se plantean ningún tipo de objetivo para salir de su zona de confort comercial.

- Los recursos humanos, tecnológicos y económicos se mantienen como principales debilidades de los productores y no presentan cambios considerables, por lo que se convierte en una prioridad impulsar estos factores de producción. Dado que la mayoría de estas Mipymes son consideradas negocios familiares, el área de recursos humanos se ve relegada a mantener el parentesco sobre los lineamientos empresariales del manejo del capital humano. Por consiguiente, no se ve necesario dotar a los trabajadores de tecnología de última generación, lo que se ve reflejado en un bajo desempeño laboral y, por ende, su competitividad se ve afectada.

\section{Referencias}

Banco Central del Ecuador (2016). Estudio sectorial para la inserción del Ecuador en los mercados mundiales. Recuperado del sitio de internet de: https:// contenido.bce.fin.ec/documentos/TLC/Sensibilidad. htm

Bayas, R., \& Cisneros, M. (2013). Análisis de la competitividad del sistema manufacturero de calzado en el cantón Cevallos (Tesis de Maestría). Universidad de las fuerzas armadas ESPE, Latacunga, Ecuador.

Bautista-Ramírez, M. L, Vargas-Hernández, J.G. (2016). Estructura empresarial y competitividad en México. Revista Libre Empresa, 13(1), 61-90 http:// dx.doi.org/10.18041/libemp.2016.v13n1.25103

Cuatrecasas, A. L. (2012). Gestión de la producción: modelos de lean management. Retrieved from http://ebookcentral.proquest.com

Cuatrecasas, A. L. (2012). Gestión económica de la producción. Retrieved from http://ebookcentral.proquest.com

Fuentes, N., Osorio, G., Mungaray, A. (2016) Capacidades intangibles para la competitividad microempresarial en México. Revista Problemas del Desarrollo, 186 (47), julio-septiembre 2016, http://probdes.iiec.unam.mx

Hall, G. (2015). Pearson's correlation coefficient. Recuperado de http:// www.hep.ph.ic.ac.uk/ hallg/UG_2015/Pearsons.pdf

Instituto de Promoción de Exportaciones e Inversiones PRO ECUADOR 
(2017). Evaluación de las exportaciones del sector cuero y calzado. Recuperado del sitio de internet: https://www.proecuador.gob.ec/exportadores/publicaciones/estadisticas-por-sector/

Ministerios de Industrias y Productividad (2017). El sector del calzado se adhiere al Acuerdo Nacional por la Producción y el Empleo. Recuperado del sitio de internet de: http://www.industrias.gob.ec/el-sector-del-calzado-se-adhiere-alacuerdo-nacional-por-la-produccion-y-el-empleo/

Montoya, L. A., Montoya, I. A., \& Castellanos, O. F. (2008). From the notion of competitiveness to the advantages of business integration. Revista Facultad de Ciencias Económicas: Investigación y Reflexión, 16(1), 59-70. Retrieved March 12, 2018, from. http://www.scielo.org.co/scielo.php?script=sci_arttext\&pid=S0121-68052008000100005\&lng=en\&t/ng=en.

Porter, M. (1990). The competitive advantage of nations, Harvard Business Review, $4^{\circ}$ trimestre, p.165. Prokopenko, J. (2009). Globalización, competitividad y estrategias de productividad: Organización Internacional del Trabajo Recuperado de https://www.oitcinterfor.org/sites/default/files/file_articulo/bol4.pdf

Ochoa, J., Parada, E., Verdugo, M. (2012) La gestión del conocimiento como factor de competitividad en las pequeñas y medianas empresas. INCEPTUM Revista de Investigación en Ciencias de la Administración. Volumen 7. Número 12. Enero-Junio 2012.

Rangel, J., Enriquez, L., Gonzales, M. (2015) La influencia de la innovación y la información financiera en la competitividad de la pequeña y mediana empresa manufacturera. Revista Internacional Administración \& Finanzas. Volumen 8. Número 2

Saavedra, M. (2012). Una propuesta para la determinación de la competitividad en la pyme latinoamericana. Pensamiento y Gestión, 33, 93-124.

Saavedra, M. (2012b). Hacia la competitividad de la Pyme latinoamericana. Macroproyecto de investigacion ALAFEC [consultado 20 Oct 2012]. Dis ponible en: http://www.alafec.unam. $m x / d o c s / m a c r o p r o y e c t o s / c o m p e t i t i v i d a d$ macro.pdf

Sarache Castro, W., \& Cárdenas Aguirre, D., \& Giraldo García, J., \& Parra Sánchez, J. (2007). Procedimiento para evaluar la estrategia de manufactura: aplicaciones en la industria metalmecánica. Cuadernos de Administración, 20 (33), 103-123.

Solleiro, J. y Castañón, R. (2005). Competitiveness and innovation systems: The challenges for Méxicos insertion in the global contex. Technovation, 45, 1059-1070.

World Economic Forum (2017). Global Competitiveness Index 2017 2018. Recuperado del sitio de internet de: http://reports.weforum.org/global-competitiveness-index-2017-2018/countryeconomy-profiles/\#economy=ECU

\section{Anexos página 23}

